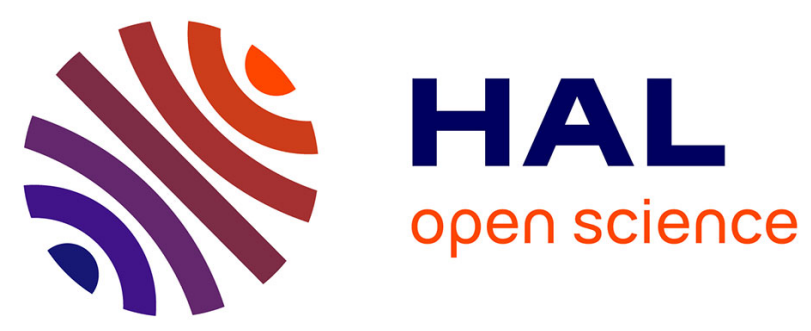

\title{
Oriented Gold Nanorods and Gold Nanorod Chains within Smectic Liquid Crystal Topological Defects
}

Brigita Rožič, Jérôme Fresnais, Celine Molinaro, Joseph Calixte, Shivakumar

Umadevi, Stephanie Lau-Truong, Nordin Felidj, Tobias Kraus, Fabrice

Charra, Vincent Dupuis, et al.

\section{To cite this version:}

Brigita Rožič, Jérôme Fresnais, Celine Molinaro, Joseph Calixte, Shivakumar Umadevi, et al.. Oriented Gold Nanorods and Gold Nanorod Chains within Smectic Liquid Crystal Topological Defects. ACS Nano, 2017, 11 (7), pp.6728-6738. 10.1021/acsnano.7b01132 . hal-01551477

\section{HAL Id: hal-01551477 \\ https://hal.sorbonne-universite.fr/hal-01551477}

Submitted on 30 Jun 2017

HAL is a multi-disciplinary open access archive for the deposit and dissemination of scientific research documents, whether they are published or not. The documents may come from teaching and research institutions in France or abroad, or from public or private research centers.
L'archive ouverte pluridisciplinaire HAL, est destinée au dépôt et à la diffusion de documents scientifiques de niveau recherche, publiés ou non, émanant des établissements d'enseignement et de recherche français ou étrangers, des laboratoires publics ou privés. 


\section{Oriented Gold Nanorods and Gold Nanorod Chains}

\section{within Smectic Liquid Crystal Topological Defects}

Brigita Rožič, ${ }^{*},+,+$ Jerome Fresnais, ${ }^{\S}$ Céline Molinaro, ${ }^{\circledR}$ Joseph Calixte, ${ }^{\ddagger}$ S. Umadevi, ${ }^{\searrow}$ Stéphanie Lau-Truong, ${ }^{\bullet}$ Nordin Félidj, $\bullet^{\bullet}$ Tobias Kraus, ${ }^{\wedge}$ Fabrice Charra, ${ }^{\circledR}$ Vincent Dupuis, ${ }^{\S}$ Torsten Hegmann, ${ }^{\mathbf{}}$ Céline Fiorini-Debuisschert, ${ }^{\alpha}$ Bruno Gallas, ${ }^{*}$ Emmanuelle Lacaze ${ }^{* *}$

"Sorbonne Université, UPMC Univ Paris 06, CNRS-UMR7588, Institut des Nano-Sciences de Paris (INSP), 4 place Jussieu, 75005 Paris, France; ${ }^{\circledR}$ Sorbonne Universités, UPMC Univ Paris 06, UMR 8234, PHENIX, F-75005 Paris, France; ' $\mathrm{S} P E C$, CEA, CNRS, Université Paris Saclay, CEA Saclay, 91191 Gif-sur-Yvette Cedex, France; DDepartment of Industrial Chemistry, Alagappa University, Karaikudi-630 002, Tamilnadu, India; •ITODYS, Université Paris Diderot, Sorbonne Paris Cité, CNRS UMR 7086, 15 rue J-A de Baif, 75205 Paris Cedex 13, France;

^INM-Leibniz Institute for New Materials, Campus D2 2, 66123 Saarbrücken, Germany;

\Liquid Crystal Institute, Kent State University Kent, OH 44242, USA 


\section{ABSTRACT}

We show that the use of oriented linear arrays of smectic A defects, the so-called smectic oily streaks, enables the orientation of gold nanorods (GNRs) for a large range of GNR diameters, ranging from 7 to $48 \mathrm{~nm}$, and for various ligands. For the small GNRs it enables oriented end-toend small chains of GNRs when the density is increased from around 2 GNRs $/ \mu m^{2}$ to around 6 GNRs $/ \mu \mathrm{m}^{2}$. We have characterized the orientation of single GNRs by spectrophotometry and Two-Photon Luminescence (TPL). A strongly anisotropic absorption of the composites and an on-off switching of GNR luminescence, both controlled by incident light polarization is observed revealing an orientation of the GNRs mostly parallel to the oily streaks. A more favorable trapping of GNRs by smectic dislocations with respect to ribbon-like defects is thus demonstrated. The dislocations appear to be localized at a specific localization, namely, the summit of rotating grain boundaries. Combining plasmonic absorption measurements, TPL measurements and simulation of the plasmonic absorption, we show that the end-to-end GNR chains are either dimers or trimers, all parallel to each other, with a small gap between the coupled GNRs, between 1 and $1.7 \mathrm{~nm}$, thus associated with a large red-shift of $110 \mathrm{~nm}$ of the longitudinal plasmonic mode. A motion of the GNRs along the dislocations appears as a necessary ingredient for the formation of end-to-end GNR chains, the gap value being driven by the balance between the attracting Van der Waals interactions and the steric repulsion between the GNRs and leading to interdigitation of the neighboring ligands. We thus obtain electromagnetic coupling of nanorods controlled by light polarization.

\section{KEYWORDS}

Smectic liquid crystal, gold nanorods, plasmon resonances, end-to-end chains, spectrophotometry, two-photon luminescence microscopy 
Nowadays assemblies of nanoparticles (NPs) for a controlled modification of their physical properties are often achieved through top-down assemblies: either NPs are built by pure lithography ${ }^{1}$ or the substrates themselves are patterned by lithography to induce assemblies of colloidal NPs templated by the substrate. ${ }^{2}$ Directed assembly of NPs is another alternative for the elaboration of materials with modified optical properties. ${ }^{3}$ Among others, this concerns metallic nanoparticles due to their unique optical properties based on localized surface plasmon (LSP) resonance characterized by a high sensitivity to the surrounding medium and to the interactions between neighboring NPs. In such a context, metallic nanorods (NRs) are particularly appealing. First, they present two different LSP modes excited by an incident light polarization perpendicular or parallel to the NR long axis and referred to as transverse or longitudinal modes, respectively. This renders both modes effective for a control of NR light absorption by incident polarized light if the NRs are oriented. ${ }^{4-6}$ Second, Gold NRs (GNRs) present strongly anisotropic field enhancement effects particularly when the longitudinal mode is excited. This can be exploited e.g. for Surface Enhanced Raman Scattering (SERS), but it was also shown to lead to strongly enhanced luminescence. ${ }^{7-10}$ If oriented NR are interesting, chains of end-to-end oriented NRs are even more appealing, due to the highly-enhanced fields created between NR extremities. This provides a promising route to nano-antennae, ${ }^{11}$ strongly signal-enhancing Raman substrates, ${ }^{12,13}$ and optical metamaterials ${ }^{14}$ from specifically organized metallic NRs. ${ }^{15}$ However, macroscopic coatings and materials inherit the microscopic anisotropic features of NRs only if the rods can be oriented along a single direction throughout the entire sample. Random distribution of NRs, as it usually occurs in a liquid, leads to random alignment and obviates their unusual anisotropic properties. A strict orientation of NRs is thus necessary to obtain activated optical properties. 
One major advantage of directed assembly is that ultra-small distances between NPs can be achieved which is rarer with top-down assemblies. ${ }^{2}$ In addition, directed assembly is based on the use of colloidal nanoparticles which quality can be enhanced with respect to nanoparticles made by lithography in terms of roughness or defect presence. However, despite the numerous works devoted to the achievement of this goal over the past decade, obtaining large scale NR assembly of controlled geometry including NR end-to-end chains remains challenging. To obtain anisotropic directed assemblies, Liquid Crystals (LCs) being intrinsically anisotropic are expected to constitute efficient matrices. In a nematic phase, orientation of NRs can be achieved, either parallel or perpendicular to the nematic director, in relation with the specificity of LC molecules' anchoring at the NR surfaces. ${ }^{16,17}$ However, NR aggregation easily occurs if the anchoring energy is too strong. ${ }^{18}$ Only specific functionalization of GNRs can lead to sufficiently weak anchoring, avoiding aggregation and allowing for reconfigurable orientation of GNRs with possible control of LSP excitation by electric fields. ${ }^{19,20}$ So far side-by-side chains have been obtained when the NR concentration is increased. ${ }^{21}$ Either for orientation or for directed assembly of GNRs, in particular for chain formation, a confinement of NRs in specific areas appears as an interesting alternative. This can be achieved in the presence of defects with singularity lines, that act as traps for nanoparticles, inducing an accumulation of the nanoparticles in and around the defect cores, reducing the molecular disorder and the free energy of the LC phase. ${ }^{22,23}$ This leads to LC phases stabilized in temperature, by the presence of nanoparticles ${ }^{24,25}$ or to the possibility of imposing electromagnetic coupling between fluorescent nanoparticles and gold nanoparticles. ${ }^{26}$ When LC defect cores are of well-defined size, shape, and orientation, they may also transfer their structural order to the nanoparticle assemblies on a large scale..$^{23,27-29,30}$ 
In the present study, we use arrays of oriented defects, the so-called oily streaks. Due to hybrid anchoring at the two interfaces (rubbed PVA (Poly(vinyl alcohol)) and air), the smectic layers become curved in flattened hemicylinders perpendicular to the anchoring on the substrate (Figure 1a). ${ }^{31-33}$ These smectic hemicylinders can be detected by optical microscopy between crossed polarizers (Figure 1c). They are formed on top of a sublayer made of perpendicular smectic layers (Figure $1 \mathrm{~b}$ ) in order to preserve the planar unidirectional anchoring on the substrate. ${ }^{34}$ This induces the presence of arrays of defects of different kinds including edge dislocations, as recently shown by a combination of x-ray diffraction and ellipsometry, ${ }^{34}$ the edge dislocations being parallel to the hemicylinder axis (OX direction - Figure 1b). Six edge dislocations of Burger vectors around 5 are formed at the summit of the rotating grain boundary (RGB, dislocations shown as orange points on Figure $1 \mathrm{~b}) ;{ }^{32}$ a small number of edge dislocations of smaller Burger vector may be also expected at the intersection between neighboring oily streaks (W, dislocations shown as blue points on Figure $1 \mathrm{~b}) ;^{35}$ a central defect is formed as well, possibly corresponding to a locally melted 2D area (blue line- Figure $1 \mathrm{~b}$ ). ${ }^{34}$

Using these oily streaks as template, we have shown that gold nanosphere (core diameter $4 \mathrm{~nm}$ ) chains are formed parallel to the dislocations. The chains being all parallel and the nanospheres being electromagnetically coupled within the chains, the plasmon absorption becomes monitored by light polarization. ${ }^{27,29}$ Single fluorescent NRs of small core diameter $(6 \mathrm{~nm})$ are oriented parallel to the dislocations, leading to a fine-tuning of the polarization of single photon emitters. ${ }^{28}$ We here report the GNR orientation induced by oily streaks for various lateral sizes of the GNRs (ranging from 7 to $48 \mathrm{~nm}$ ) and the formation of end-to-end chains of oriented GNRs for the smallest sizes. We study the corresponding anisotropy of light absorption and luminescence controlled by light polarization. Using the simulation of light absorption, we 
evidence the mechanism of end-to-end chain formation driven by the confinement in the dislocations and we estimate the gap between the GNR extremities in the chains. This allows for the demonstration of a large electromagnetic coupling between GNRs controlled by the light polarization.

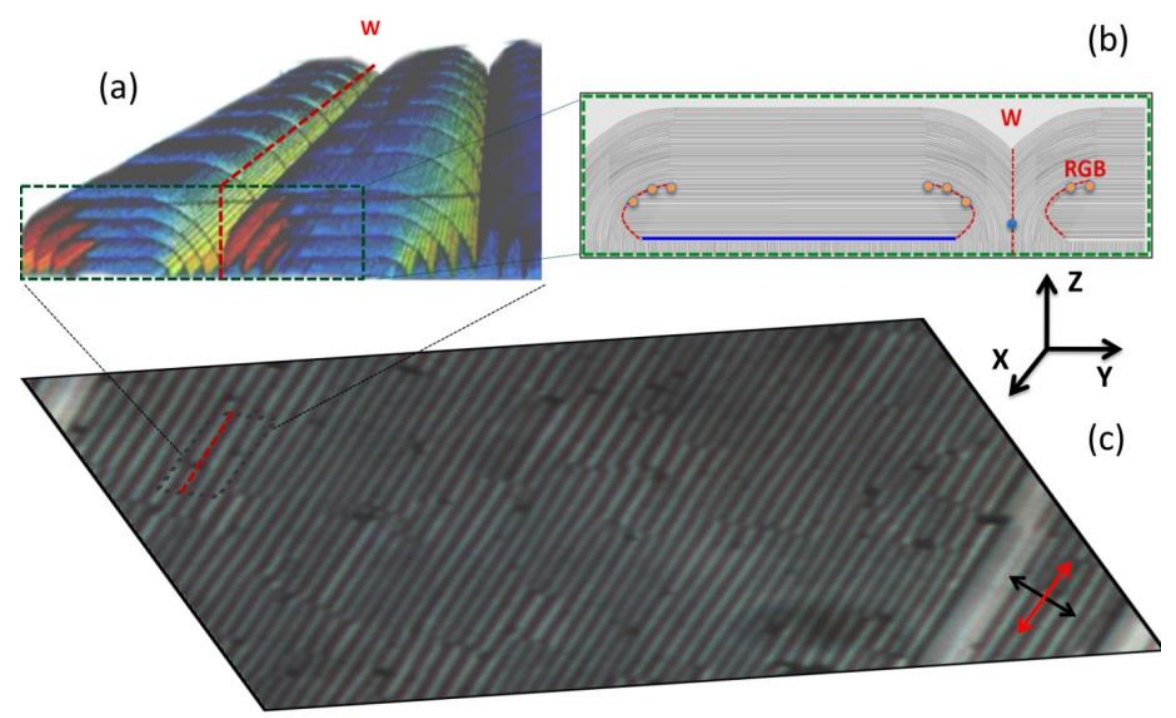

Figure 1. (a) Schematic presentation of the oily streaks structure where only few smectic layers are represented, organized in parallel hemicylinders containing curvature walls (W), oriented along OX. (b) Detail in side-view (in the $(Y, Z)$ plane) of the smectic layers stacked in flattened hemicylinders with grain boundaries in red and blue, the curvature wall $W$, the inner rotating grain boundary (RGB) and the central grain boundary, including dispersed edge dislocations (orange and blue points). All smectic layers are represented for a typical thickness of $230 \mathrm{~nm}^{34}$ (c) Polarized optical microscopy image of oily streaks observed from the top of a $150 \mathrm{~nm}$-thick sample $(20 \mu \mathrm{m} \times 10 \mu \mathrm{m})$. Each line corresponds to one hemicylinder. 


\section{RESULTS AND DISCUSSION}

\section{Small GNR concentration}

\section{GNR1 LSP}

Figure $2 \mathrm{a}$ displays the extinction spectrum of a $50 \mu \mathrm{m} \times 50 \mu \mathrm{m}$ area of 8CB (4-n-octyl-4'-cyanobiphenyl) LC oily streaks in the presence of GNR1s (the small GNRs of diameter of $7 \mathrm{~nm}$ and a length of $25 \mathrm{~nm}$ (see Section Material and Methods)) for a composite film of thickness $150 \mathrm{~nm}$ and a GNR1 density around 2 GNR1s per $\mu \mathrm{m}^{2}$. This extinction spectrum is obtained with nonpolarized incident light excitation and displays the two LSP bands of GNR1s, as expected. The wavelengths associated with the two LSP bands are found to be $512 \pm 5 \mathrm{~nm}$ and $801 \pm 7 \mathrm{~nm}$. These values are close to the values in toluene (512 and $798 \mathrm{~nm}$, see Fig. S1a), where the optical index is $\mathrm{n}=1.49$, very close to the $8 \mathrm{CB}$ ordinary index $\left(\mathrm{n}_{\mathrm{o}}=1.52\right)$, but lower than the extraordinary index $\left(\mathrm{n}_{\mathrm{e}}=1.67\right) .512$ and $801 \mathrm{~nm}$ correspond to the average values of 17 measurements made on 17 oily streak zones $(50 \mu \mathrm{m}$ x $50 \mu \mathrm{m})$ of similar thickness in the same sample, as checked by optical microscopy.

The intensity of the first band appears abnormally high with respect to the second band. This most likely indicates a difference in thickness between the LC films with GNR1s and the reference without GNR1s that has been used to extract the GNR1 extinction. Simulations of the influence of the LC thickness indeed show that the first band intensity is much more sensitive to LC thickness than the second band, however without significant modification of the band wavelength (See SI). It can be also noticed that the second band on Figure $2 \mathrm{a}$ is broader than the one measured in solution (Full Width at Half Maximum $(\mathrm{FWHM})=138 \mathrm{~nm}$ in average versus $115 \mathrm{~nm}$ ). The broadening may indicate the formation of aggregates in the LC film; however, they 
are clearly in a smaller number than on the rubbed PVA substrate without LC, as shown by the larger FWHM of $161 \mathrm{~nm}$ for this latter sample (Figure S2), also confirmed by TEM picture on carbon grids (Figure S1c).

Figure $2 \mathrm{~b}-\mathrm{c}$ presents the LSP extinction for two perpendicular polarizations parallel to the oily steaks for Figure $2 \mathrm{~b}$ and perpendicular for Figure 2c, respectively. An anisotropic GNR1 organization is revealed. When the incident light is polarized parallel to the oily streaks, the longitudinal LSP band is detected (Figure 2b). Its average over the same 17 zones appears at approximately the same wavelength as for the second band of Figure $2 \mathrm{a}, \lambda_{2}=810 \pm 10 \mathrm{~nm}$, which is also close to the second band of GNR1s dispersed in pure toluene (798 nm, see Fig. S1a). For perpendicular polarization the extinction measurements show domination of the transverse LSP band (Figure 2c), which average wavelength is again close to the one obtained with nonpolarized light $\left(\lambda_{1}=512 \pm 5 \mathrm{~nm}\right.$, Figure $\left.2 \mathrm{a}\right)$ and to the one of GNRs in toluene $\left(\lambda_{1}=512 \mathrm{~nm}\right.$, Fig. S1a): $\lambda_{1}=514 \pm 9 \mathrm{~nm}$. It corresponds to the average over the same 17 zones. Some signal above $850 \mathrm{~nm}$, still observed in the plot shown in Figure 2c, may be related to the small number of aggregates discussed above, this latter hypothesis being supported by the red-shift of this band with respect to isolated GNR1s in toluene. The appearance of this band at $850 \mathrm{~nm}$ for perpendicular polarization may be indicative of a random orientation of the aggregates. This is in contrast with the majority of GNR1s, the observation of mainly the longitudinal LSP band for the polarization parallel to the oily streaks and the transverse LSP band for the perpendicular polarization allowing the conclusion that the GNR1s are predominantly oriented parallel to the oily streaks. Since the two LSP bands, now selected by incident light polarization, appear to correspond to the ones of isolated GNR1s in toluene, without any electromagnetic coupling 
between them, the concentration may be small enough to consider the GNR1s as isolated from each other.

As a result of the imposed orientation of GNR1s along oily streaks, the light absorption wavelength can be varied by $296 \mathrm{~nm}$ (from $514 \mathrm{~nm}$ to $810 \mathrm{~nm}$ ) when the incident light polarization is varied by $90^{\circ}$. We have also varied the lateral size of GNR, from $7 \mathrm{~nm}$ (GNR1) to $48 \mathrm{~nm}$ (GNR2), which leads to very similar results (see Fig. S3). This shows that the upper limit for an induced orientation of single GNRs is beyond a diameter of $48 \mathrm{~nm}$. However, the large size of GNR2s may induce some GNR organization within the aggregates also formed in the LC film, as shown by the two small bands at large wavelength for perpendicular polarization (Figure S3c), but still without any orientation of the aggregates induced by the oily streaks.

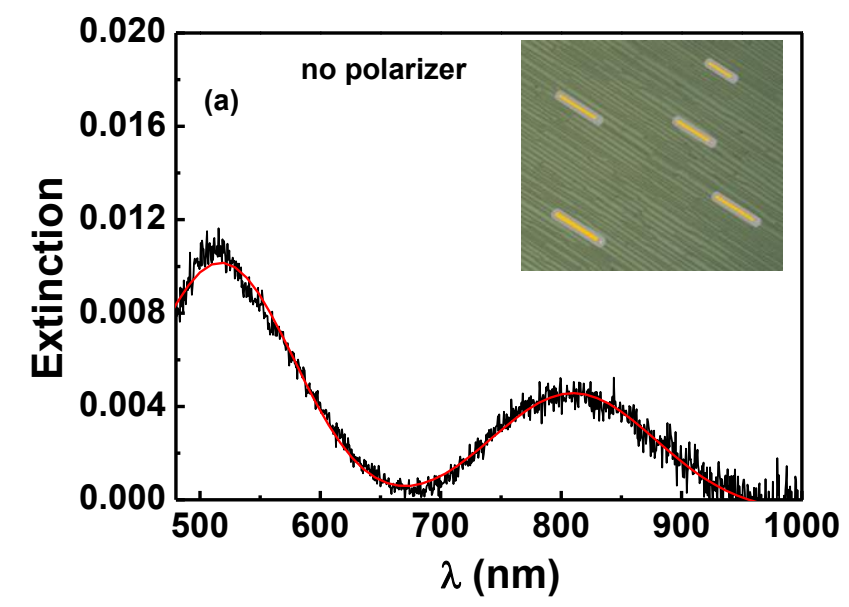



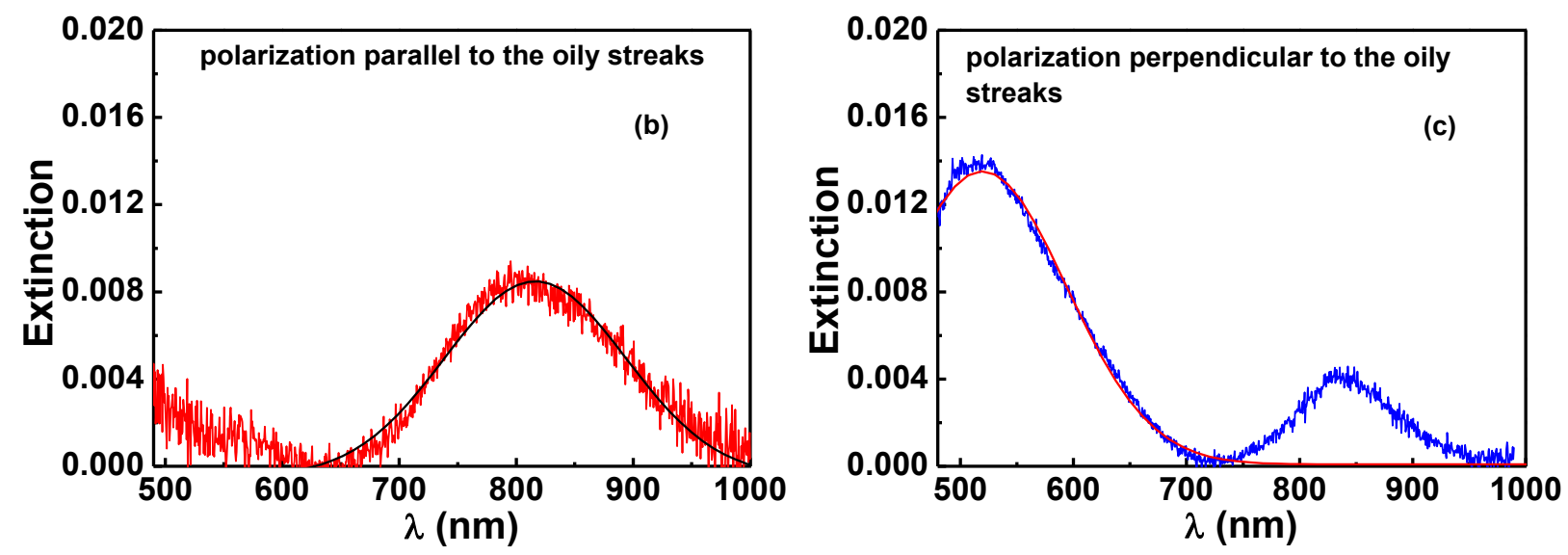

Figure 2. Extinction measurements of GNR1s/8CB (around $2 \mathrm{GNRs} / \mathrm{\mu m}^{2}, 8 \mathrm{CB}$ thickness of $150 \mathrm{~nm}$ ) samples deposited on PVA polymer with (a) non-polarized incident light, and with an incident light polarized (b) parallel and (c) perpendicular to the oily streaks. The solid lines correspond to Gaussian fits allowing for the extraction of the plasmon band wavelength. The inset on (a) corresponds to a scheme of the related GNR1s in LC deposited on the rubbed PVA-glass substrate, in relation with the oily streaks.

\section{GNR1 TPL}

The two-photon luminescence (TPL) imaging has been used to characterize the GNRs organization in composites made of GNRs and nematic LCs. ${ }^{19,20}$ Here, we have measured a GNR1/LC sample with similar GNR density and LC thickness than for the sample in Figure 2. The TPL signal of GNRs is well known to be maximal when the GNRs are excited at their longitudinal plasmon resonance, i.e. for an excitation wavelength $\lambda_{\mathrm{exc}}=\lambda_{\mathrm{LSP}}$ and a polarization along the GNRs long axis: ITPL is proportional to $\cos ^{4}(\theta)$, with $\theta$, the angle between the incident light polarization and the GNR longitudinal axis..$^{10,36}$ 
Typical TPL images are shown in Figure 3a-3b. With the excitation laser power kept constant, the excitation wavelength was first varied between 780 and $950 \mathrm{~nm}$. It was found that GNR1s emit the strongest luminescence for the excitation wavelength $\lambda_{\text {exc }}=820 \mathrm{~nm}$, in line with the longitudinal LSP wavelength previously evidenced through the extinction spectrum of a similar sample (Figure 2b). The most striking feature resulting from Figure 3 is that, for a given sample area, the TPL images happen to be fundamentally dependent on the incident laser polarization, either parallel (Figure 3a) or perpendicular (Figure 3b) to the oily streaks. This is a clear sign for a preferential orientation of the GNR1s along the oily streaks. Indeed, the TPL becomes quasiswitched on/off when going from an incident polarization parallel to perpendicular to the oily streaks, except for a few intense spots (marked by a green circle in Figures 3a and 3b, respectively) for which the signal is measured and determined to remain unchanged when the polarization is varied. These latter TPL spots may correspond to aggregates already detected in the LSP data (Figure 2c).

(a) Polarization parallel to the oily streaks

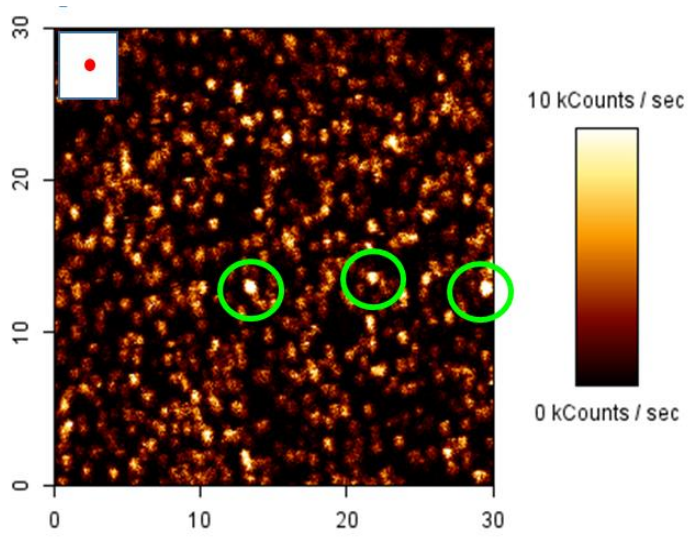

(b) Polarization perpendicular to the oily streaks

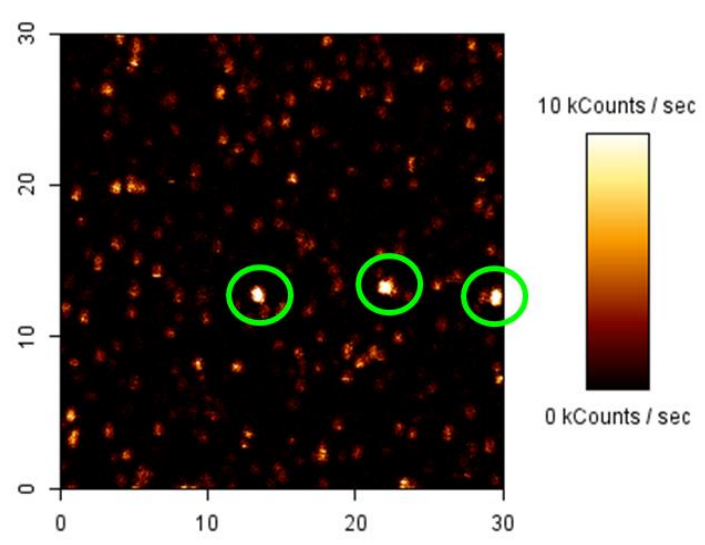




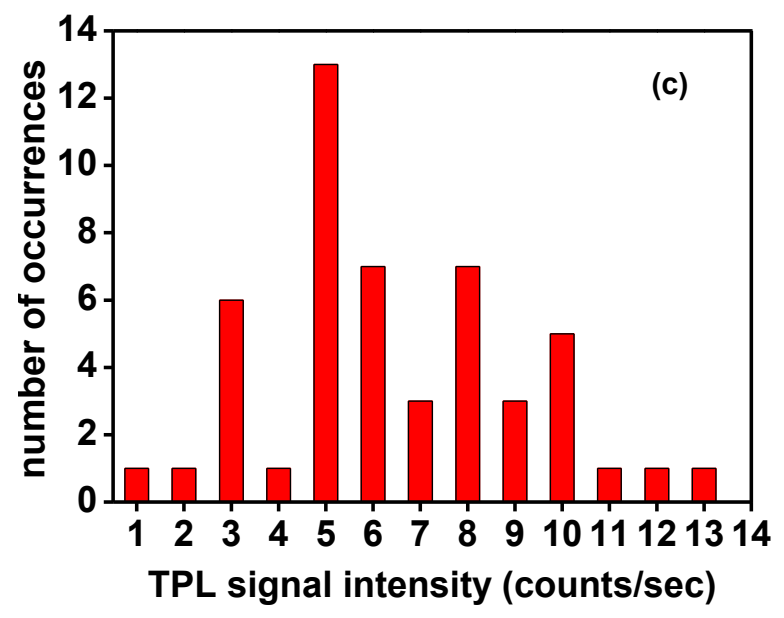

Figure 3. Two-photon luminescence (TPL) of a sample of GNR1s in 8CB (around 2 GNRs $/ \mu^{2}$ on average, $8 \mathrm{CB}$ thickness of $\left.150 \mathrm{~nm}\right)$. The laser excitation and average power were respectively $820 \mathrm{~nm}$ (longitudinal GNR1 LSPR) and $500 \mu \mathrm{W}$ average power. The same zone was raster scanned with a $600 \mathrm{~nm}$ beam waist (lateral extension schematized as a red disk in insert) using either an excitation polarization along (a) or perpendicular to (b) the oily streaks. (c) Intensity histogram for the spots oriented mainly parallel to the oily streaks (85\% of the spots).

A statistical analysis of the TPL spots performed using the same images obtained with a polarization either parallel or perpendicular to the stripes, shows that if we do not consider the little amount of totally isotropic aggregates, $85 \%$ of the spots are oriented parallel to the oily streaks with an angle better than $45^{\circ}$, with around half of the total amount at an angle better than $30^{\circ}$. This is in good agreement with a well-defined GNR1 orientation parallel to the oily streaks, already revealed in Figure $2 \mathrm{~b}-\mathrm{c}$. The remaining $15 \%$ of the spots may correspond to non-oriented GNR1s. 
We observed roughly $720 \mathrm{TPL}$ spots per $900 \mu \mathrm{m}^{2}$ in Figure 3a. The same density of spot has been obtained on 5 different areas of the sample. It is important to note that the analysis of the lateral extension of each TPL spot was shown to be fully consistent with the $\mathrm{w}=600 \mathrm{~nm}$ exciting laser beam waist raster scanned on the sample for TPL imaging: $\mathrm{I}_{\mathrm{TPL}} \alpha \mathrm{I}_{\mathrm{exc}}^{2} \alpha$ exp-4(r$\left.\mathrm{r}_{0}\right)^{2} / \mathrm{w}^{2}$ (see Fig. S4). The intensity histogram for the TPL spots polarized along the oily streaks (Figure 3c) can be extracted from Figure 3a, suggesting a maximum that is slightly smaller than $6 \mathrm{kcps} / \mathrm{s}$ (Figure 3c). In a complementary analysis, the signal obtained with a single similar GNR excited in the same conditions was measured to be $3 \mathrm{kcps} / \mathrm{s}$, allowing for an estimation of the average number of GNR1s per TPL spot. The histogram of Figure 3c is consistent with a large number of TPL spots associated with two GNR1s. However, it is slightly asymmetric towards the large number of GNR. Taking into account this asymmetry and the presence of some aggregates, we obtain an average number between 2 and 3 GNR1s per TPL spot, mostly well oriented to the oily streaks as shown above, associated with an average density around 2 GNR1s per $\mu \mathrm{m}^{2}$. In case of homogeneously dispersed GNR1s, this would have led to $0.8 \mathrm{GNR} /$ laser spot and to a quasi-homogeneous illumination in the Figure $3 a-3 b$, in contrast with the observation. This slightly non-homogeneous dispersion of GNR1s in the smectic film may be initiated by an inhomogeneous evaporation of the solvent leading to the formation in the smectic film of areas more concentrated in GNRs. However, as shown by the LSPR results, together with the observation of a TPL maximum occurring for an excitation at $820 \mathrm{~nm}$, the GNR1s remain isolated from each other in the most areas associated with the TPL spots, without any significant electromagnetic coupling. 


\section{Large GNR concentration}

\section{GNR1}

With the increase of the surface density of GNR1s by a factor 2.7, leading to a GNR1 density around $6 \mathrm{GNR} 1 / \mu \mathrm{m}^{2}$ (LC thickness of $250 \mathrm{~nm}$ ), the two plasmon bands are again obtained using unpolarized light (Figure 4a), with wavelength maxima at $512 \mathrm{~nm}( \pm 5 \mathrm{~nm})$ and $922 \mathrm{~nm}( \pm 8 \mathrm{~nm})$ respectively - average over 18 measured zones. The first wavelength maximum is found close to the one of GNRs1 in toluene, but the second one is red-shifted by about $120 \mathrm{~nm}$ with respect to toluene and $110 \mathrm{~nm}$ with respect to the sample of Figure 2. For the polarization parallel to the oily streaks, mostly the second extinction peak is still obtained, however red-shifted by about $120 \mathrm{~nm}\left(\lambda_{2}=922 \pm 7 \mathrm{~nm}\right.$; average over the same 18 measured zones) in comparison to the longitudinal LSP mode of GNR1s in toluene (Fig. S1a), which is consistent with the results obtained without polarization (Figure 4a). When the incident light is polarized perpendicular to the oily streaks, the first extinction peak dominates. It appears at $\lambda_{1}=510 \pm 8 \mathrm{~nm}$ (average over the same 18 measured zones). On Figure 4c a small signal around $850 \mathrm{~nm}$ may be associated again with the presence of a small number of non-oriented aggregates, already shown in Figure 3. However, the observation of the transverse band mainly observed with perpendicular polarization and the longitudinal band with parallel polarization shows that GNR1s are in majority oriented parallel to the oily streaks. In addition, the observation of the red-shift of the longitudinal band with respect to Figure $2 \mathrm{~b}$ indicates an end-to-end electromagnetic coupling between GNR1s, associated with the formation of end-to-end chains of GNR1s parallel to the oily streaks. ${ }^{37,38}$ It results in a hybridation between the longitudinal LSP mode of GNRs in close vicinity. ${ }^{39}$ This shows that there are linear trapping areas in the oily streaks that not only orient but also localize GNRs, such that when the concentration is increased, an electromagnetic 
coupling occurs between the ends of the GNRs, leading to large modifications of the LSP properties.
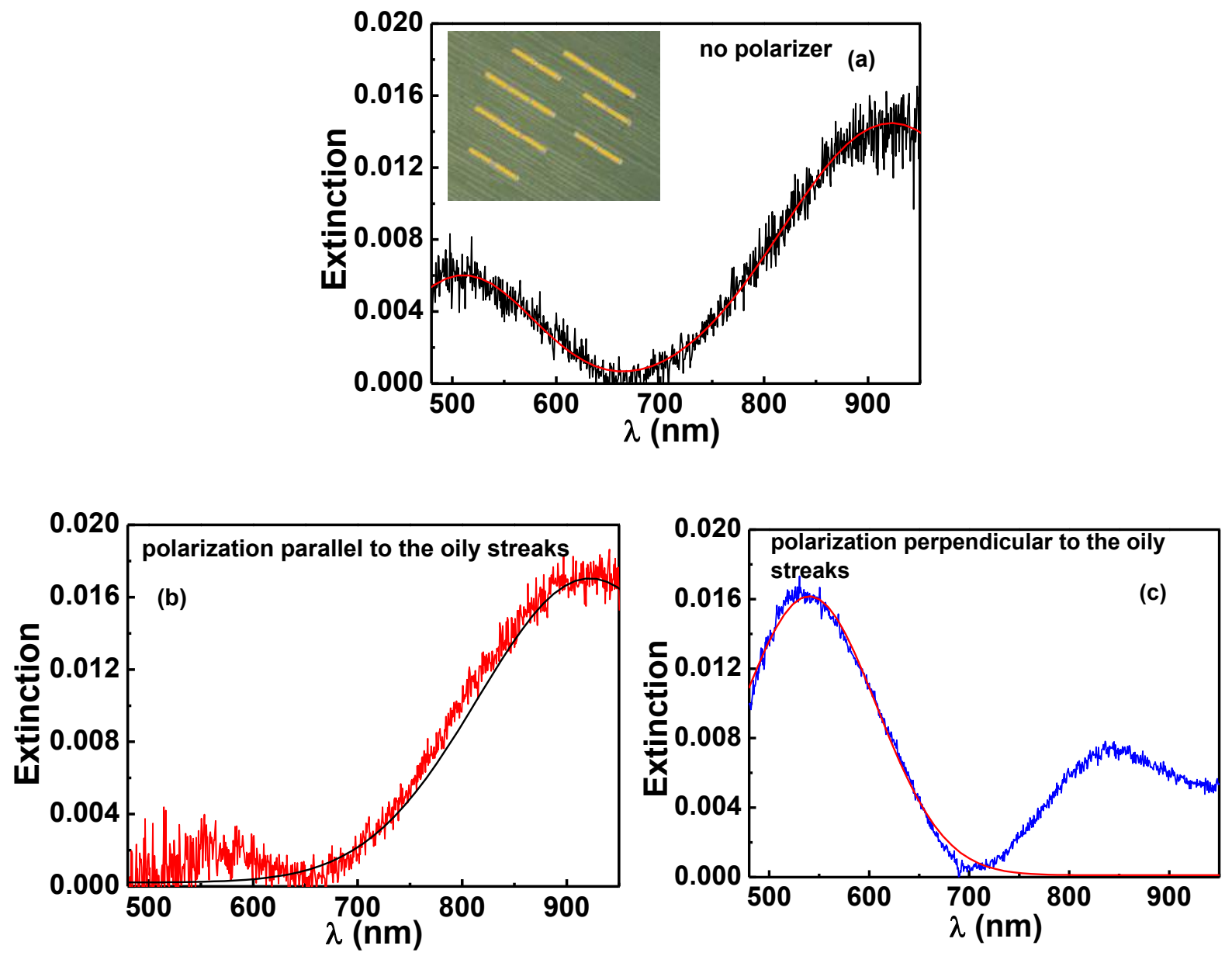

Figure 4. Extinction measurements of GNR1s/8CB (around $6 \mathrm{GNRs} / \mu^{2} \mathrm{~m}^{2}, 8 \mathrm{CB}$ thickness of $250 \mathrm{~nm}$ ) samples deposited on PVA polymer with an incident light (a) non-polarized and polarized (b) parallel and (c) perpendicular to the oily streaks. Solid lines correspond to Gaussian fits allowing for the extraction of the plasmon band wavelength. The inset corresponds to a scheme of the expected orientation and organization of the GNR1s in LC, in relation with the oily streaks. 
The large red-shift of the longitudinal band depends on the gap between neighboring GNR1s and on the number of GNR1s involved in the chains. In order to gain a better insight into such coupling effects, we have conducted simulations of the absorption of GNRs embedded in a liquid crystal.

\section{Simulations}

The calculated absorption cross-section for different numbers $\mathrm{N}$ of end-to-end coupled GNRs (multimers), with a gap value of $1 \mathrm{~nm}$ between adjacent GNRs, is presented in Figure 5a. The exciting electric field was polarized at $60^{\circ}$ from the long axis to simulate the case of multimers disordered in a liquid crystal environment. Two absorption bands are observed. The first one near $540 \mathrm{~nm}$ for isolated GNRs corresponds to the excitation of the transverse plasmon mode. The second one near $820 \mathrm{~nm}$ for isolated GNRs, corresponds to the excitation of the longitudinal plasmon mode and is close to the longitudinal mode found for GNR1s in Figure 3. This mode red-shifts when the number $\mathrm{N}$ of GNRs increases in each multimer. A red-shift towards $935 \mathrm{~nm}$ is obtained for trimers, close to the experimental results shown on Figure 4 and it reaches a value of $970 \mathrm{~nm}$ in pentamers (Figure 5a). The red-shift does not increase linearly with $\mathrm{N}$ as already described in the literature (Figure 5a-inset). ${ }^{37,38,40-44}$ 

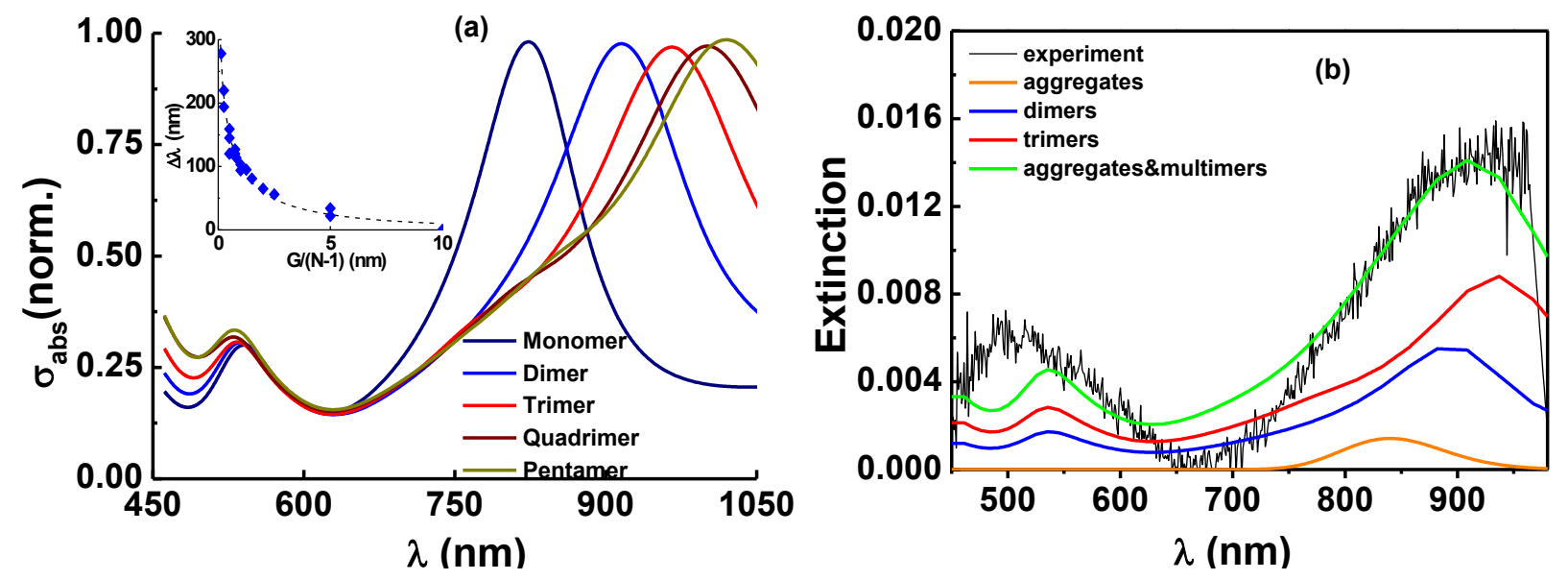

Figure 5. (a) Calculated absorption cross-section of end-to-end coupled GNRs with a gap value of $1 \mathrm{~nm}$. The relative shift of the LSP for different types of multimers (from dimers to octamers) and different gap values is presented in the inset (blue symbols). The dotted line presents the fit to a plasmon ruler equation (see text). (b) Adjustment of the experimental absorption extracted from Figure $4 \mathrm{c}$ to the superposition of the absorption of dimmers and trimers (47\% and 53\%) with $1.7 \mathrm{~nm}$ gap and the phenomenological absorption of aggregates.

When the gap decreases, the red-shift increases. Different configurations (gap value, number of multimer) have been calculated. The inset summarizes the evolution of the LSPR shift $\Delta \lambda$ as a function of $\mathrm{G} /(\mathrm{N}-1)$ with $\mathrm{N}$ the number of monomer and $\mathrm{G}$ the gap value in nanometers. This evolution can be reproduced by a general plasmon ruler equation modified from that of Ref. 38 to take into account the number of monomers and can be cast in:

$$
\Delta \lambda=\lambda_{\mathrm{o}} \beta \exp -\left[(1 / \tau)(\mathrm{G} / \mathrm{L}(\mathrm{N}-1))^{1 / 3}\right]
$$


with $\lambda_{0}$ the position of the LSPR of isolated GNR and L the length of the GNRs. $\beta=0.9$ and the decay rate $\tau=0.17$ are fitting parameters. The values of $\beta$ and $\tau$ are in good agreement with the values found in the literature. ${ }^{38}$

To get insight in the origin of the absorption observed in the film with large density of nanorods, Figure $5 \mathrm{~b}$ presents the unpolarized absorption measured in Figure 4a together with a model based on the numerical calculations. Using the plasmon ruler of Figure 5a, we obtain for a longitudinal band at $922 \mathrm{~nm}$ a gap of $2 \mathrm{~nm}$ for trimers and $1 \mathrm{~nm}$ for dimers. However the experimental data don't fit with a unique kind of multimers, neither trimers nor dimers, even if we take into account the presence of the aggregates already evidenced on Figure 2 (We reproduced this absorption band associated with aggregates with a Gaussian line shape and added this absorption band in our model - orange line on Figure 5b). This is because of the too small width of the trimer (green curve on Figure 5b) or dimer (braun curve on Figure 5b) longitudinal band. As a result, if we want to avoid the presence of quadrimers, a majority of trimers would require a gap around $1.7 \mathrm{~nm}$, the best superimposition with experiment data leading to $53 \%$ of trimers and $47 \%$ of dimers. As shown by Figure $5 \mathrm{~b}$ the experimental data are then correctly reproduced by the simulations (red curve on Figure 5b). The assumption of dominating dimers with a gap $1 \mathrm{~nm}$ also correctly fit the data, leading to $66 \%$ of dimers and $34 \%$ of trimers and we can not discriminate between the two assumtions, gap of $1 \mathrm{~nm}$ or gap of $1.7 \mathrm{~nm}$, the real structure possibly being an intermediate one. The fitting procedure also led to a negligible amount of single GNR1s.

\section{Mechanism of orientation and formation of GNR chains in LC}


A strict orientation of NRs had already been shown for semi-conducting NRs, the so-called dotin-rods, of diameter $7 \mathrm{~nm}$ and length $23 \mathrm{~nm}$, both similar to GNR1 s. ${ }^{28}$ Therefore, the control of the polarization of emitted light by single photon emitters had become possible. ${ }^{28}$ We now demonstrate that gold NRs, GNR1s, of same size but with different ligands, made of liquid crystal molecules ${ }^{29}$ instead of TOP (trioctylphosphine) and ODPA (octadecylphosphonic acid) ${ }^{25}$ can be oriented as well, along a unique direction for a given sample, the direction being perpendicular to the substrate rubbing. This now not only allows a controlled excitation of GNR plasmon resonance absorption by incident light polarization (varying from the wavelength 538 $\mathrm{nm}$ to $814 \mathrm{~nm}$ when the polarization is rotated from perpendicular to parallel to the oily streaks Figure 2), but also allows the control of sample luminescence (Figure 3). The control of luminescence of GNRs is rare since intense luminescence occurs mostly for GNRs of small size and GNRs happen to be isotropically distributed in most samples (for instance in solutions or polymeric matrices). We here obtain an on-off control of the luminescence, driven by the incident polarization, due to the preferential anisotropic organization of the GNRs oriented parallel to the oily streaks. We anticipate this induced orientation to be associated with the presence of linear defects oriented parallel to the hemicylinder axis (Figure 1). As shown in Figure 1, large ribbons (blue line on Figure 1) corresponding to 2D defects have been evidenced in oily streaks, coexisting with linear dislocations parallel to the hemicylinder axis, thus perpendicular to the substrate rubbing (points on Figure 1) ${ }^{34}$ In the 2D ribbons of width larger than $400 \mathrm{~nm}$, no well-defined orientation of the GNRs is expected, except if the 2D ribbon indeed corresponds to a nematic area. In this latter case, a GNR orientation parallel to the nematic director, thus perpendicular to the oily streaks direction would preferably occur in contrast to our observation. ${ }^{11,30}$ Our results thus show that linear dislocations, strictly parallel to 
oily streak direction, are better traps than the ribbon-like defects. A size matching between defect cores and GNR inducing an orientation of the GNR parallel to the defect cores, also detected through their TPL signal, has already been evidenced for cholesteric defects of toron structure. ${ }^{22}$ We expect a size matching between the GNR1 width $(7 \mathrm{~nm})$ and the size of the smectic dislocation cores dispersed within the oily streaks, ranging from 3 to $15 \mathrm{~nm} \cdot{ }^{28,45}$ The size matching may promote GNR1 trapping inside the linear dislocation cores with an orientation parallel to the dislocations, the volume of disordered liquid crystal expelled by the GNR1s being thus maximum. ${ }^{28}$ If we consider elementary smectic dislocations, their energy per unit of length has been measured in free standing smectic films to be $0.5 \mathrm{kT} . \AA \AA-1 .{ }^{46-48} \mathrm{~A}$ single GNR1 of length 1 $=25 \mathrm{~nm}$, trapped in the dislocation core and parallel to its axis, allows the decrease of the liquid crystal disorder energy by $125 \mathrm{kT}$, leading to a significant advantage for the liquid crystal film, due to the elongated shape of GNRs. GNR1s, once trapped and oriented parallel to the smectic dislocations may be particularly well-stabilized. The GNR1 diameter of $7 \mathrm{~nm}$ being larger than the core of elementary dislocations, around $3 \mathrm{~nm},{ }^{45}$ a localization in the dislocations at the summit of the rotating grain boundary, of Burger vector around 5, thus of core size of the order of $15 \mathrm{~nm}$ (orange points on Figure 1b) may be preferred. Our results even demonstrate that orientation of GNRs still holds for GNRs of diameter as large as $48 \mathrm{~nm}$ (GNR2s) (Fig. S3). These GNRs are considerably larger than the dislocations expected in oily streaks. A drawback can be the disorder induced around the dislocations despite the advantage to locate GNRs in the dislocation cores and in the elastically distorted areas around the cores. As a result, for large GNRs, like GNR2s, the 3 parallel dislocations of Burger vector of the order of 5, separated from each other by roughly $30 \mathrm{~nm}$, at the summit of the rotating grain boundary (blue points on Figure 1) may consist in the most favorable trapping sites with possibly one single GNR2 over two 
parallel dislocations. Using GNR2s, we have shown that for an aspect ratio of nanorods around 3, the upper limit of GNR diameter for a well-defined induced orientation in the oily streaks is above $48 \mathrm{~nm}$. We expect that such orientation would hold for any aspect ratio, and also for GNRs of diameter as large as $100 \mathrm{~nm}$, in relation with the presence of the three consecutive dislocations trapping one single GNR.

The comparison between the data shown in Figure 2 and Figure 4 demonstrates that a concentration increase by a factor 2.7 causes a transformation from an ensemble of single oriented GNR1s (sample of Figure 2 and Figure 3) to an ensemble of coupled oriented GNR1s with end-to-end geometry. This formation of oriented GNR1 multimers is obviously related to the strong GNR1 confinement induced by the LC dislocation. However, with such a small GNR1 density, some motion of the GNR1s along the dislocations may be required as well to explain the experimental observations.

We can thus estimate the diffusion coefficient of GNR1s in the disordered 8CB molecules which would constitute the core of the smectic dislocations. The Brownian diffusion coefficient of anisotropic particles along the longitudinal axis is expected to be the one of a sphere of similar volume divided by 2.93 , a factor defined by the GNR1 aspect ratio ${ }^{50,51}$. Using the viscosity of the isotropic $8 \mathrm{CB}^{52}$, the GNR1 diffusion coefficient is found to be $4.8 \times 10^{5} \mathrm{~nm}^{2} \mathrm{~s}^{-1}$. As a result of Brownian motion, the time necessary to obtain a mean square displacement of $100 \mathrm{~nm}$ is of the order of $0.01 \mathrm{~s}$ for a 1 dimensional diffusion along the dislocations. We consequently expect a significant motion of GNR1s along the dislocation, which has already been observed with fluorescent nanorods of size similar to the GNR1 size, always parallel to the dislocations. ${ }^{28}$ As a result, for a sufficiently high concentration, the motion may only stop when one GNR1 meets its neighboring GNR1. The gap value between them would be the result of the balance between the 
attracting Van der Waals interactions and the steric repulsion between the GNR1s leading to possible interdigitation of the ligands of neighboring GNR1s. ${ }^{29}$ This is confirmed by the recent results obtained for nanospheres of diameter $4 \mathrm{~nm}$ covered by dodecanethiols which appear to be organized in chains in the same oily streaks, the corresponding gaps varying between 0.6 and 1.9 nm. ${ }^{29}$ GNR1 ligands are made of liquid crystal attached to siloxane, of length being roughly 1.5

times the dodecanthiol one (Figure 6) ${ }^{46}$ Comparison with nanosphere chains thus suggests gap values between 0.9 and $2.8 \mathrm{~nm}$ between coupled GNR1s. However, an easier interdigitation between the ligands may occur for GNR1 ligands with respect to dodecanethiols ligands of nanospheres due to the well-known smaller number of ligands at the extremities of the nanorods with respect to their edges. ${ }^{4,13}$ It thus appears reasonable to consider a gap between GNR1s of around $1.5 \mathrm{~nm}$, Figure 5a insert shows that a gap of $2 \mathrm{~nm}$ corresponds to the experimental maximum of the longitudinal band of trimers and a gap of $1 \mathrm{~nm}$ corresponds to the one of dimers. However the experimental band appears too large to be associated with a unique kind of multimers (Figure 4 and Figure 5b). Accordingly, using a mixture between dimers and trimers of GNR1s separated by a gap of $1.7 \mathrm{~nm}$, we can theoretically reproduce the experimental results of Figure $4 a$ (Figure 5b). An alternative explanation for Figure 4 would be a mixture between dimers, trimers or quadrimers. In order to discriminate between the different possibilities a precise knowledge of the ligand density and structure would be necessary. However, we can also discriminate by examining the formation of multimers that could occur within a model of smectic hemicylinders with 7 dislocations like the one shown on Figure 1, whose structure has been extracted from a combination of X-ray and ellipsometry measurements. ${ }^{34}$ We make the assumption that the GNR1 accumulate in areas of surface equal or smaller than $0.36 \mu \mathrm{m}^{2}$, the size of the laser beam waist used in the TPL experiments. This is an 
extrapolation for a concentration around 6 GNR1s per $\mu \mathrm{m}^{2}$ of the TPL observations for a concentration around 2 GNR1s per $\mu \mathrm{m}^{2}$ showing TPL spots associated with an average number of GNRs between 2 and 3 GNR1s per TPL spot. We would expect for the concentration around 6 GNR1s per $\mu \mathrm{m}^{2}$ between 5.5 and 8 GNR1s in the area equal or smaller than $0.36 \mu \mathrm{m}^{2}$. Let us consider that all 7 dislocations are equally favorable for the GNR1 trapping. For the sample with a concentration around 6 GNR1s per $\mu \mathrm{m}^{2}$ (Figure 4), the LC thickness was around $250 \mathrm{~nm}$. According to the known curve of the hemicylinder period as a function of thickness, ${ }^{32}$ the hemicylinder period is of the order $700 \mathrm{~nm}$. In an area of $0.36 \mu \mathrm{m}^{2}$, where the GNR1s would accumulate, 6 dislocations may be available and we expect between 5.5 and 8 GNR1s accumulated in the area, leading to roughly one GNR1 per dislocation. For the samples with a concentration around 2 GNR1s per $\mu \mathrm{m}^{2}$ (Figure 2 and Figure 3), the thickness was $150 \mathrm{~nm}$, yielding a period of the order of $500 \mathrm{~nm}$. The same reasoning would lead to roughly $1 / 3$ of GNR1 in average per dislocation. In both cases, the GNR1 would be spatially isolated on the average and quasi-no electromagnetic coupling is expected in contradiction with the observations of Figure 4. To induce more than 1 GNR1 in each dislocation and allow for coupling between at least two GNR1s in majority for the concentration around 6 GNR1s per $\mu \mathrm{m}^{2}$, the size of the area where GNR1s accumulate has to be not larger than $0.076-0.16 \mu \mathrm{m}^{2}$, allowing for at least 2 GNR1s in average in each dislocation. Since the sample of concentration around 2 GNR1s per $\mu \mathrm{m}^{2}$ (Figure 2 and Figure 3), does not contain coupled GNR1s, the size of this area also must be not smaller than $0.028-0.046 \mu \mathrm{m}^{2}$. Such small values for the area where GNR1s accumulate would lead to a number of 3 GNR1s in average per dislocation for the sample with the highest concentration. As a consequence, only dimers or trimers may be expected for this sample. In addition, the formation of coupled GNR1s for the large concentration would also require a 
motion of GNR1s along the dislocations of at least 100 or $400 \mathrm{~nm}$, depending on the exact value of GNR1 density. As shown above, this motion is likely to occur when considering the diffusion coefficient value of GNR1s in a disordered 8CB. Two features would thus allow for the observed multimer formation, a localization of the GNR1s in small spot sizes, most probably related to solvent inhomogeneous evaporation, together with a motion of GNR1s within the dislocations, of at least $100-400 \mathrm{~nm}$. For the concentration here considered, around 6 GNR1s per $\mu \mathrm{m}^{2}$ this would lead to a mixture of dimers and trimers, the gap value being between 1 and $1.7 \mathrm{~nm}$, as demonstrated by the simulations of Figure $5 b$.

Oily streaks providing a large density of linear and oriented defects with singularity lines, we can finally easily create end-to-end chains of GNRs, even if only small amounts of GNRs are used. An increase of GNR concentration may allow for an increase above 3 of the number of GNR1s involved in the chains, the spacing between GNRs being expected to remain monitored by the ligand interdigitation. If all dislocations consist in efficient traps, the GNR1 localization is mostly expected in the dislocations at the summit of the RGB shown on Figure 1. Since the dislocations are separated by $30 \mathrm{~nm}$, a lateral electromagnetic coupling between the chains can be avoided only if the GNR width is small enough. For GNRs of width small enough, formation of closed packed GNRs occurs in an end-to-end geometry and may induce intense hot spots between the GNR1s. If the chains are all parallel to each other, the electromagnetic coupling between GNR1s become activated by light polarization and the formation of the expected hot spots as well. 


\section{CONCLUSION}

In conclusion, the use of oriented linear arrays of smectic A defects, the so-called oily streaks, enables the orientation of gold nanorods (GNRs) for a large range of diameter, ranging from 7 to $48 \mathrm{~nm}$, but also, for smaller GNRs, the GNR1s (diameter $7 \mathrm{~nm}$ ), it enables GNR organization within oriented end-to-end small chains when the density is increased from around 2 GNR1s $/ \mu \mathrm{m}^{2}$ towards around $6 \mathrm{GNR} 1 \mathrm{~s} / \mu \mathrm{m}^{2}$. This leads to strongly anisotropic LSP absorption monitored by incident light polarization, that could vary from $530 \mathrm{~nm}$ to $920 \mathrm{~nm}$ when end-toend coupled GNRs are concerned. In addition, we show for GNR1s that two-photon luminescence signal becomes monitored as well by incident light polarization. We can conclude that GNRs are preferentially trapped in linear dislocations, in particular dispersed at the summit of the rotating grain boundaries present in oily streaks but not in the $2 \mathrm{D}$ ribbon-like defect. Combining plasmonic absorption measurements, TPL measurements and simulation of the plasmonic absorption, we show that the formation of end-to-end coupled GNRs is also the result of a local increase of GNRs concentration, possibly related to inhomogeneous solvent evaporation, combined with a motion of GNRs along the dislocations. The final gap between coupled GNRs is thus driven by the balance between Van der Waals attraction and steric repulsion between the GNR1s and leads to ligand interdigitation, with gaps between coupled GNRs between 1 and $1.7 \mathrm{~nm} \mathrm{~nm}$ associated with the formation of a mixture of dimers and trimers. We thus obtain highly coupled GNRs of electromagnetic coupling controlled by light polarization. 


\section{MATERIALS AND METHODS}

In order to study a directed assembly of GNRs, pure smectic liquid crystal (LC) 8CB (4-n-octyl4'-cyano-biphenyl) and composites made of GNRs embedded in 8CB have been investigated. We deposited both materials on thin glass substrates covered by a rubbed polymer (PVA), which induces planar anchoring of the LC director parallel to the rubbing direction. We prepared a solution of pure $8 \mathrm{CB}$ and a solution of $\mathrm{GNRs} / 8 \mathrm{CB}$ in toluene and carefully deposited them on the rubbed PVA-glass substrates by using a drop-casting method at room temperature.

We investigated two kinds of GNRs with different dimensions and ligands of different nature. We first focused on GNRs with a diameter of $7 \mathrm{~nm}$ and a length of $25 \mathrm{~nm}$ (GNR1). The aspect ratio is about 3.5, and was measured using electron microscopy. GNR1s were chemically functionalized with thermotropic nematic liquid crystals (Figure 6), which work as stabilizing ligands, ${ }^{46}$ and they were dispersed in toluene (concentration around $8 \times 10^{13} \mathrm{GNRs} / \mathrm{L}$ as estimated by luminescence data). Second, larger GNRs (GNR2) with dimensions of $48 \mathrm{~nm}$ in diameter and $145 \mathrm{~nm}$ in length (i.e. GNRs with an aspect ratio around 3), have been investigated, corresponding to a concentration in gold atoms of $0.1667 \mathrm{mg} / \mathrm{ml}$, as measured by Rutherford Back Scattering (RBS) experiments. After deposition of a droplet on a silicon substrate, no gold nanosphere was evidenced by Scanning Electron Microscopy, thus leading to a concentration of $3 \times 10^{13}$ GNRs/L. GNR2s were synthesized by using hexadecyltrimethylammonium bromide (CTAB) surfactant with aromatic additives and they were dispersed in pure water. ${ }^{53}$ The change of solvent, from water to toluene, has been made in a few steps, ultimately leading to a coating of the GNR2s by polydimethylsiloxane (PDMS). ${ }^{54}$ The extinction spectra of both GNRs in toluene display two maxima, corresponding to the transverse and longitudinal surface plasmon resonance, situated at $512 \mathrm{~nm}$ and $798 \mathrm{~nm}$ for GNR1s (Fig. S1a), and at $535 \mathrm{~nm}$ and $820 \mathrm{~nm}$ in 
the case of GNR2s (Fig. S1b). These values agree with those found in the literature for nanorods of similar aspect ratio. ${ }^{46,53,55}$

Solutions of GNR1s and LC in toluene were prepared. Two mixtures with respectively $8 \times 10^{13}$ GNR1s/L, $2 \times 10^{-2} \mathrm{M} 8 \mathrm{CB}$ in toluene and $1.3 \times 10^{14} \mathrm{GNR} 1 \mathrm{~s} / \mathrm{L}, 2 \times 10^{-2} \mathrm{M} 8 \mathrm{CB}$ were prepared and carefully drop-casted on $18 \mathrm{~nm} \times 18 \mathrm{~nm}$ large glass substrates with rubbed PVA. A large number of samples was prepared. We varied not only the amount of GNRs, but also the thickness of the 8CB layer atop. 150 and $250 \mathrm{~nm}$ thick films with an average GNR1 surface density varying between around 2 and around 6 GNR1s per $\mu \mathrm{m}^{2}$ were obtained. After deposition of a droplet of solution, samples were left to dry for about 20 minutes at room temperature to allow the slow evaporation of toluene and the creation of oily streaks. We checked by optical microscopy (Figure 1c) that the oily streak texture remained largely unchanged after incorporation of the GNRs.

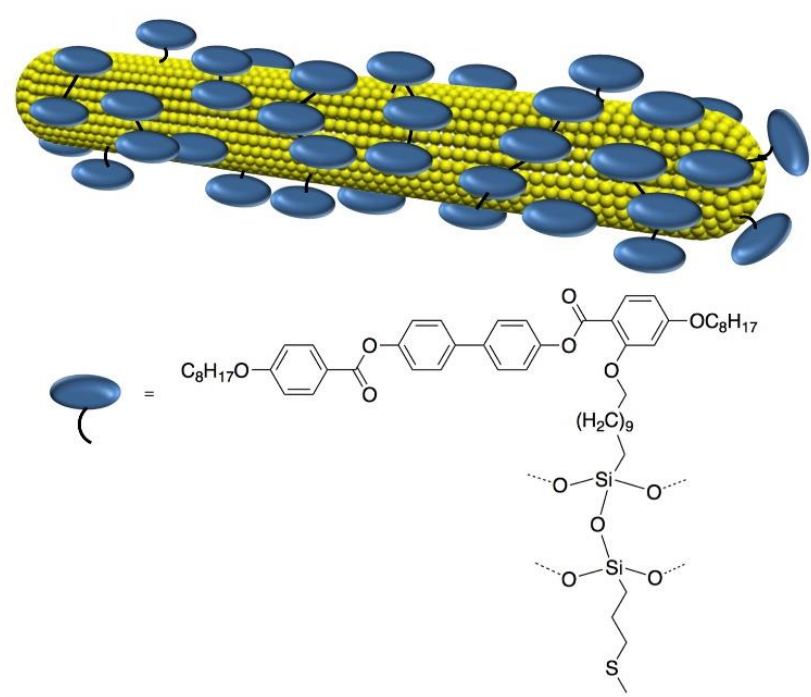

Figure 6. Schematic representation of the side-on nematic liquid crystal-coated GNRs1. Silane-conjugation among the liquid crystal thiols (i.e. the LC-thiols are linked to one another) as described in ref. [46] produces thermally and chemically robust gold nanorods. 
For GNR2s, the preparation was similar. Mixtures of $3 \times 10^{13} \mathrm{GNRs} / \mathrm{L}$ and $3 \times 10^{-2} \mathrm{M} 8 \mathrm{CB}$ were drop-casted on a rubbed PVA substrate. We prepared samples of LC thickness of $200 \mathrm{~nm}$, leading to an average surface density of 0.2 GNR2s per $\mu \mathrm{m}^{2}$. Again, we observed no change in the oily streaks by the presence of GNR, as shown by polarized optical microscopy.

The organization of the GNRs within the LC defects was revealed through measurements of the LSP of the GNRs in LC. Measurements were performed by using a LOT Oriel MS260i spectrophotometer. The spectrometer was coupled with an optical microscope (Olympus BX 51) driven in transmission mode via an optical fiber, which can probe an area of $50 \mu \mathrm{m} \times 50 \mu \mathrm{m}$ of the sample. The transmission spectra $\mathrm{T}$ were normalized to reference measurements $\mathrm{T}_{\text {ref }}$ made of samples of pure $8 \mathrm{CB}$ containing oily streaks of similar thickness but without nanorods. The spectra are presented as extinction spectra defined as $\mathrm{Ext}=-\log \left(\mathrm{T} / \mathrm{T}_{\mathrm{ref}}\right)$. To ensure a proper normalization of the measured data, it is necessary that the reference sample has exactly the same thickness as the sample containing the gold NRs. The procedure used to determine the thickness of the samples (oily streak period together with measurements of Newton tints ${ }^{34}$ ) yields an uncertainty of $50 \mathrm{~nm}$ (between 20 and 30\%) on the actual thickness, also related to the inhomogeneity of thickness on the area of $50 \mu \mathrm{m} \times 50 \mu \mathrm{m}$ where the measurements are performed. Since we use small concentrations of GNR, this uncertainty on the thicknesses induces uncertainties on the values of the extinctions at the wavelength positions of the LSP and marginally on the positions themselves. With the range of thickness used here we can estimate the uncertainty on the values of extinctions of the longitudinal mode to be of $10 \%$ (see S5). It is not so true however for the transverse mode (see S5), which explains some unexpected variation of the transverse mode amplitude with respect to the longitudinal one, the effect being even worse with varying polarized light, since the optical indices are expected to vary with light 
polarization. We measured the optical properties on many different areas of each sample, the presented LSP wavelength values being the averaged band maxima of all measurements (between 17 and 20 areas, depending on the samples) made with unpolarized light, and with two perpendicular light polarizations, parallel and perpendicular to oily streaks. The exact LSP positions were obtained by analyzing the experimental data using One- and Two-peak Gaussian fitting functions.

The two-photon luminescence (TPL) properties of the GNRs in LC were measured using a femto-second Titanium-Sapphire laser source (Tsunami, SpectraPhysics), delivering 100 fs pulses with an $80 \mathrm{MHz}$ repetition rate over the spectral range covering 770 to $950 \mathrm{~nm}$. The laser, which polarization and power can be controlled, is focused onto the sample using a $40 \times$, NA (Numerical Aperture) 0.95 microscope objective in an inverted optical microscope configuration. The sample is then raster scanned while the TPL is recorded leading to the construction of a point-by-point image. The emitted TPL is collected through the same microscope objective as for excitation and separated from the incident light by a dichroic mirror (SemRock FF735-Di670$25 \times 36$ ). The signal is then sent either to a channel plate multiplier working in the photon counting mode (Perkin Elmer MP-993-CL) or to a spectrometer coupled to a CCD camera (Andor DU401-BR-DD) for detailed study of the emission spectra.

The absorption of GNR and chains of GNRs in LC has been calculated using Finite Element Methods (HFSS by ANSYS). The optical constants of gold were modeled from those of bulk gold. ${ }^{55}$ The optical constants were decomposed in an interband contribution and a Drude term. The interband contribution was that of bulk gold but the relaxation time of the Drude term was divided by a factor of three to account for the broadening of the LSP observed in toluene. The decrease of the relaxation time partly originates from scattering of the free electrons at the 
surface of the GNR, ${ }^{56}$ but it may also partly take into account some size dispersion of the GNRs. The GNRs were modeled as cylinders of diameter $7 \mathrm{~nm}$ with rounded ends for a total length of $25 \mathrm{~nm}$. The optical properties were calculated using a unit cell of dimensions $1 \mu \mathrm{m} \times 1 \mu \mathrm{m} \times 1$ $\mu \mathrm{m}$ with radiating boundaries. The dimension of the unit cell allowed all the near-field contribution to be exhausted before reaching the radiating surface of the unit cell. The optical constant of the ambient medium in the unit cell was set to the weighted average of the ordinary and extraordinary optical constants of $8 \mathrm{CB}$. The illumination was provided by a polarized plane wave impinging on the GNR perpendicularly to its long axis. The absorption cross-section $\sigma_{\mathrm{abs}}$ was obtained from the ohmic losses in the GNRs calculated from the current distributions $\overrightarrow{J_{\text {vol }}}$ in the volume $V$ of each GNR summed over all nanorods as: $\sigma_{\mathrm{abs}}=\sum_{\mathrm{GNR}}\left(\int_{\mathrm{V}} \overrightarrow{\mathrm{J}_{\mathrm{vol}}} \cdot \overrightarrow{\mathrm{E}} \cdot \mathrm{dV}\right)$. This geometry yielded a good agreement between measured and calculated absorption of isolated nanorods in toluene (see S6).

\section{ASSOCIATED CONTENT}

\section{Supporting Information}

The Supporting Information is available free of charge on the ACS Publications website at DOI: ...

Supplementary information of gold nanorods, including TEM and SEM images, spectrophotometry of GNRs in toluene and of GNR2s in LC and some additional simulations.

\section{AUTHOR INFORMATION}




\section{Corresponding Authors}

* E-mail: brigita.rozic@,ijs.si

* E-mail: emmanuelle.lacaze@insp.jussieu.fr

\section{Present Addresses}

† Jožef Stefan Institute, Jamova cesta 39, 1001 Ljubljana, Slovenia

\section{Author Contributions}

The manuscript was written through contributions of all authors. All authors have given approval to the final version of the manuscript.

\section{Notes}

The authors declare no competing financial interest.

\section{ACKNOWLEDGMENT}

BR acknowledges the Labex MATISSE for the support and funding of postdoctoral project. The work was also partly supported by the Partner University Fund program funded by French embassy in USA. TH acknowledges financial support from the Ohio Third Frontier (OTF) program for Ohio Research Scholars "Research Cluster on Surfaces in Advanced Materials". The authors acknowledge help of G. Guida at LEME (U. Paris 10) for help with FEM calculations and help of S. Royer, E. Briand and L. Becerra, respectively for plasmonic measurements, RBS measurements and use of INSP clean room. 


\section{REFERENCES}

1. Shawrav, M. M.; Taus, P.; Wanzenboeck, H. D.; Schinnerl, M.; Stöger-Pollach, M.; S. Schwarz, S.; Steiger-Thirsfeld, A.; Bertagnolli, E. Highly Conductive and Pure Gold Nanostructures Grown by Electron Beam Induced Deposition. Sci. Rep. 2016, 6, 34003.

2. Flauraud, V.; Mastrangeli, M.; Bernasconi, G. D.; Butet, J.; Alexander, D. T. L.; Shahrabi, E.; Martin, O. J. F.; Brugger, J. Nanoscale Topographical Control of Capillary Assembly of Nanoparticles. Nat. Nanotechnol. 2016, 12, 73-80.

3. Grzelczak, M.; Vermant, J.; Furst, E. M., Liz-Marzán, L. M. Directed Self-Assembly of Nanoparticles. ACS Nano 2010, 4, 3591-3605.

4. Pérez-Juste, J.; Pastoriza-Santos, I.; Liz-Marzán, L. M.; Mulvaney, P. Gold Nanorods: Synthesis, Characterization and Applications. Coord. Chem. Rev. 2005, 249, 1870-1901.

5. Sharma, V.; Park, K.; Srinivasarao, M. Colloidal Dispersion of Gold Nanorods: Historical Background, Optical Properties, Seed-Mediated Synthesis, Shape Separation and SelfAssembly. Materials Science and Engineering $R$ 2009, 65, 1-38.

6. Huang, X.; Neretina, S.; El-Sayed, M. A. Gold Nanorods: From Synthesis and Properties to Biological and Biomedical Applications. Adv. Mater. 2009, 21, 4880-4910.

7. Mohamed, M. B.; Volkov, V.; Link, S.; El-Sayed, M. A. The 'Lightning' Gold Nanorods: Fluorescence Enhancement of Over a Million Compared to the Gold Metal. Chem. Phys. Lett. 2000, 317, 517-523. 
8. Yorulmaz, M.; Khatua, S.; Zijlstra, P.; Gaiduk, A.; Orrit, M. Luminescence Quantum Yield of Single Gold Nanorods. Nano Lett. 2012, 12, 4385-4391.

9. Horneber, A.; Braun, K.; Rogalski, J.; Leiderer, P.; Meixner, A. J.; Zhang, D. Nonlinear Optical Imaging of Single Plasmonic Nanoparticles with $30 \mathrm{~nm}$ Resolution. Phys. Chem. Chem. Phys. 2015, 17, 21288-21293.

10. Molinaro, C.; Harfouch, Y. E.; Palleau, E.; Eloi, F.; Marguet, S.; Douillard, L.; Charra, F.; Fiorini-Debuisschert, C. Two-Photon Luminescence of Single Colloidal Gold Nanorods: Revealing the Origin of Plasmon Relaxation in Small Nanocrystals. J. Phys. Chem. C 2016, $120,23136-23143$.

11. Kosako, T.; Kadoya, T.; Hofmann, H. F. Directional Control of Light by a Nano-Optical Yagi-Uda Antenna. Nat. Photonics 2010, 4, 312-315.

12. Le Ru, E. C.; Etchegoin, P. G. Principles of Surface-Enhanced Raman Spectroscopy and Related Plasmonic Effects; Elsevier Science: Great Britain, 2008.

13. Haidar, I.; Aubard, J.; Lévi, G.; Lau-Truong, S.; Mouton, L.; Neuville, D. R.; Félidj, N.; Boubekeur-Lecaque, L. Design of Stable Plasmonic Dimers in Solution: Importance of Nanorods Aging and Acidic Medium. J. Phys. Chem. C 2015, 119, 23149-23158.

14. Ni, X.; Wong, Z. J.; Mrejen, M.; Wang, Y.; Zhang, X. An Ultrathin Invisibility Skin Cloak for Visible Light. Science 2015, 349, 1310-1314. 
15. Li, I.; Suen, J. J.-Y.; Prince1, E.; Larin, E. M.; Klinkova, A.; Therien-Aubin, H.; Zhu, S.; Yang, B.; Helmy, A. S.; Lavrentovich, O. D.; Kumacheva, E. Colloidal Cholesteric Liquid Crystal in Spherical Confinement. Nat. Commun. 2016, 7, 12520.

16. Lynch, M. D.; Patrick, D. L. Organizing Carbon Nanotubes with Liquid Crystals. Nano Lett. 2002, 2, 1197-1201.

17. Burylov, S. V.; Raikher, Y. L. On the Orientation of an Anisometric Particle Suspended in a Bulk Uniform Nematic. Phys. Lett. A 1990, 149, 279-283.

18. Blanc, C.; Coursault, D.; Lacaze, E. Ordering Nano- and Microparticles Assemblies with Liquid Crystals. Liq. Cryst. Rev. 2013, 1, 83-109.

19. Liu, Q.; Yuan, Y.; Smalyukh I. I. Electrically and Optically Tunable Plasmonic Guest-Host Liquid Crystals with Long-Range Ordered Nanoparticles. Nano Lett. 2014, 14, 4071-4077.

20. Mundoor, H.; Smalyukh, I. I. Mesostructured Composite Materials with Electrically Tunable Upconverting Properties. Small 2015, 11, 5572-5580.

21. Zhang, Y.; Liu, Q.; Mundoor, H.; Zuan, Y.; Smalyukh, I. I. Metal Nanoparticle Dispersion, Alignment, and Assembly in Nematic Liquid Crystals for Applications in Switchable Plasmonic Color Filters and E-Polarizers. ACS Nano 2015, 9, 3097-3108.

22. Evans, J. S.; Ackerman, P. J.; Broer, D. J.; van de Lagemaat, J.; Smalyukh, I. I. Optical Generation, Templating, and Polymerization of Three-Dimensional Arrays of Liquid-Crystal Defects Decorated by Plasmonic Nanoparticles. Phys. Rev. E 2013, 87, 032503. 
23. Milette, J.; Relaix, S.; Lavigne, C.; Toader, V.; Cowling, S. J.; Saez, I. M; Lennox, R. B.; Goodby, J. W.; Reven, L. Reversible Long-Range Patterning of Gold Nanoparticles by Smectic Liquid Crystals. Soft Matter 2012, 8, 6593-6598.

24. Yoshida, H.; Tanaka, Y.; Kawamoto, K.; Kubo, H.; Tsuda, T.; Fujii, A.; Kuwabata, S.; Kikuchi, H.; Ozaki, M. Nanoparticle-Stabilized Cholesteric Blue Phases. Appl. Phys. Express 2009, 2, 121501.

25. Karatairi, E.; Rožič, B.; Kutnjak, Z.; Tzitzios, V.; Nounesis, G.; Cordoyiannis, G.; Thoen, J.; Glorieux, C.; Kralj, S. Nanoparticle-Induced Widening of the Temperature Range of LiquidCrystalline Blue Phases. Phys. Rev. E 2010, 81, 041703.

26. Ackerman, P. J.; Mundoor, H.; Smalyukh, I. I.; van de Lagemaat, J. Plasmon-Exciton Interactions Probed Using Spatial Coentrapment of Nanoparticles by Topological Singularities. ACS Nano, 2015, 9, 12392-12400.

27. Coursault, D.; Grand, J.; Zappone, B.; Ayeb, H.; Lévi, G.; Félidj, N.; Lacaze, E. Linear SelfAssembly of Nanoparticles Within Liquid Crystal Defect Arrays. Adv. Mater. 2012, 24, $1461-1465$.

28. Pelliser, L.; Manceau, M.; Lethiec, C.; Coursault, D.; Vezzoli, S.; Leménager, G.; Coolen, L.; DeVittorio, M.; Pisanello, F.; Carbone, L.; Maitre, A.; Bramati, A.; Lacaze, E. Alignment of Rod-Shaped Single-Photon Emitters Driven by Line Defects in Liquid Crystals. Adv. Funct. Mater. 2015, 25, 1719-1726.

29. Coursault, D.; Zappone, B.; Coati, A.; Boulaoued, A.; Pelliser, L.; Limagne, D.; Boudet, N.; Ibrahim, B. H.; de Martino, A.; Alba, M.; Goldmann, M.; Garreau, Y.; Gallas, B.; Lacaze, E. 
Tailoring Anisotropic Interactions between Soft Nanospheres Using Dense Arrays of Smectic Liquid Crystal Edge Dislocations. ACS Nano 2015, 9, 11678-11689.

30. Gryn, I.; Lacaze, E.; Carbone, L.; Giocondo, M.; Zappone, B. Electric-Field-Controlled Alignment of Rod-Shaped Fluorescent Nanocrystals in Smectic Liquid Crystal Defect Arrays. Adv. Funct. Mater. 2016, 26, 7122-7131.

31. Michel, J.; Lacaze, E.; Alba, M.; de Boissieu, M.; Gailhanou M.; Goldmann, M. Optical Gratings Formed in Thin Smectic Films Frustrated on a Single Crystalline Substrate. Phys. Rev. E 2004, 70, 011709.

32. Michel, J.; Lacaze, E.; Goldmann, M.; de Boissieu, M.; Gailhanou, M.; Alba, M. Structure of Smectic Defect Cores: An X-ray Study of 8CB Liquid Crystal Ultra-Thin Films. Phys. Rev. Lett. 2006, 96, 027803.

33. Zappone, B.; Lacaze, E. Surface-Frustrated Periodic Textures of Smectic-A Liquid Crystals on Crystalline Surfaces. Phys. Rev. E 2008, 78, 061704.

34. Coursault, D.; Zappone, B.; Coati, A.; Boulaoued, A.; Pelliser, L.; Limagne, D.; Boudet, N.; Ibrahim, B. H.; de Martino, A.; Alba, M.; Goldmann, M.; Garreau, Y.; Gallas, B.; Lacaze, E. Self-Organized Arrays of Dislocations in Thin Smectic Liquid Crystal Films. Soft Matter 2016, 12, 678-688.

35. Williams, C; Kleman, M. Dislocations, Grain Boundaries and Focal Conics in Smectics A. J. de Physique 1975, 36, C1: 315-320. 
36. Zijlstra, P.; Chon, J. W.; Gu, M. Five-Dimensional Optical Recording Mediated by Surface Plasmons in Gold Nanorods. Nature 2009, 459, 410-413.

37. Funston, A. M.; Novo, C.; Davis, T. J.; Mulvaney, P. Plasmon Coupling of Gold Nanorods at Short Distances and in Different Geometries. Nano Lett. 2009, 9, 1651-1658.

38. Woo, K. C.; Shao, L.; Chen, H.; Liang, Y.; Wang, J.; Lin, H.-Q. Universal Scaling and Fano Resonance in the Plasmon Coupling between Gold Nanorods. ACS Nano 2011, 5, 59765986.

39. Gomez, D.; Teo, Z.; Altissimo, M.; Davis, T.; Earl, S.; Roberts, A. The Dark Side of Plasmonics. Nano Lett. 2013, 13, 3722-3728.

40. Girard, C. Near Fields in Nanostructures. Rep. Prog. Phys. 2005, 68, 1883-1933.

41. Pelton, M.; Aizpurua, J.; Bryant, G. Metal-Nanoparticle Plasmonics. Laser Photonics Rev. 2008, 2, 136-159.

42. Yockell-Lelièvre, H.; Gingras, D.; Vallée, R.; Ritcey, A. M. Coupling of Localized Surface Plasmon Resonance in Self-Organized Polystyrene-Capped Gold Nanoparticle Films. J. Phys. Chem. C 2009, 113, 21293-21302.

43. Khlebtsov, N. G.; Dykman, L. A. Optical Properties and Biomedical Applications of Plasmonic Nanoparticles. J. Quant. Spectrosc. Radiat. Transfer 2010, 111, 1-35.

44. Tripathy, S.; Marty, R.; Lin, V. K.; Teo, S. L.; Ye, E.; Arbouet, A.; Saviot, L.; Girard, C.; Han, M. Y.; Mlayah, A. Acousto-Plasmonic and Surface-Enhanced Raman Scattering Properties of Coupled Gold Nanospheres/Nanodisk Trimers. Nano Lett. 2011, 11, 431-437. 
45. Kleman, M. Points, Lines, and Walls: In Liquid Crystals, Magnetic systems, and Various Ordered Media; John Wiley\&Sons Inc.: New York, 1977.

46. Umadevi, S; Feng, X.; Hegmann, T. Large Area Self-Assembly of Nematic Liquid-CrystalFunctionalized Gold Nanorods. Adv. Funct. Mater. 2013, 23, 1393-1403.

47. Feng, X.; Sosa-Vargas, L.; Umadevi, S.; Mori, T.; Shimizu, Y.; Hegmann, T. Discotic Liquid Crystal-Functionalized Gold Nanorods: 2- and 3D Self-Assembly and Macroscopic Alignment as well as Increased Charge Carrier Mobility in Hexagonal Columnar Liquid Crystal Hosts Affected by Molecular Packing and $\pi-\pi$ Interactions. Adv. Funct. Mater. 2013, $23,1393-1403$.

48. Shukla, R. K.; Feng, X.; Umadevi, S.; Hegmann, T.; Haase, W. Influence of Different Amount of Functionalized Bulky Gold Nanorods Dopant on the Electrooptical, Dielectric and Optical Properties of the FLC Host. Chem. Phys. Lett. 2014, 599, 80-85.

49. Lee, E.; Xia, Y.; Ferrier, R. C.; Kim, H.-N.; Gharbi, M. A.; Stebe, K. J.; Kamien, R. D.; Composto, R. J.; Yang, S. Fine Golden Rings: Tunable Surface Plasmon Resonance from Assembled Nanorods in Topological Defects of Liquid Crystals. Adv. Mater. 2016, 28, $2731-2736$.

50. Perrin F. Brownian Motion of an Anisotropic Particle. J. de Phys. et Rad. V 1934, 497.

51. Perrin F. Brownian Motion of an Anisotropic Particle. J. de Phys. et Rad. VII 1936, 1.

52. Schneider, F. Measurement of the Viscosity Coefficient $\eta_{3}$ in Free-Standing Smectic Films. Phys. Rev. E 2006, 74, 021709. 
53. Ye, X.; Jin, L.; Caglayan, H.; Chen, J.; Xing, G.; Zheng, C.; Doan-Nguyen, V.; Kang, Y.; Engheta, N.; Kagan, C. R.; Murray, C. B. Improved Size-Tunable Synthesis of Monodisperse Gold Nanorods through the Use of Aromatic Additives. ACS Nano 2012, 3, 2804-2817.

54. Berry, K. R.; Russell, A. G.; Blake, P. A.; Roper, D. K. Gold Nanoparticles Reduced in situ and Dispersed in Polymer Thin Films: Optical and Thermal Properties. Nanotechnology 2012, 23, 375703.

55. Vigdermann, L.; Khanal, B. P.; Zubarev, E. R. Functional Gold Nanorods: Synthesis, SelfAssembly, and Sensing Applications. Adv. Mater. 2012, 24, 4811-4841.

56. Park, K.; Drummy, L. F.; Wadams, R. C.; Koerner, H.; Nepal, D.; Fabris, L.; Vaia, R. A. Growth Mechanism of Gold Nanorods. Chem. Mater. 2013, 25, 555-563.

\section{$\underline{\text { TOC graphic: }}$}

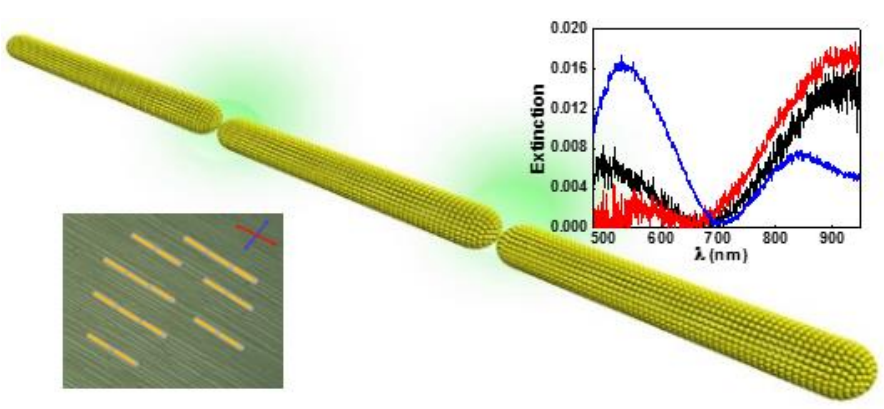

